\title{
La evaluación de cursos de formación online: el caso ISTAS
}

\author{
Evaluation of Online Training. ISTAS Case
}

\author{
Javier Rodríguez-Santero \\ Universidad de Sevilla. España. \\ jarosa@us.es \\ Juan Jesús Torres-Gordillo \\ Universidad de Sevilla. España. \\ juanj@us.es
}

\begin{abstract}
Resumen
Este artículo pretende exponer un ejemplo real de cómo pueden evaluarse cursos de formación online, dedicándose especial atención a la transferencia del aprendizaje. La investigación se ha llevado a cabo mediante un estudio de encuesta aplicado a 471 participantes de toda España que han recibido los cursos de formación online desarrollados por la fundación ISTAS (Instituto Sindical de Trabajo, Ambiente y Salud). Se ha efectuado un análisis psicométrico, descriptivo y correlacional. La fiabilidad del cuestionario se determinó mediante Alfa de Cronbach y la validez de constructo a través del Análisis Factorial Exploratorio (AFE) por componentes principales (ACP). El análisis de los datos, cuantitativo y cualitativo, nos ha permitido constatar buenos resultados en todos los factores analizados (diseño pedagógico, actuación del tutor, transferencia del aprendizaje logrado en el curso, temporalización y diseño del entorno virtual). Se logra también distinguir diferentes niveles de transferencia en función de distintos criterios (antigüedad en el sector laboral, tipología de empresa y motivo por el cual se realizó el curso). La transferencia fue moderada, y se materializa en la aplicación de conocimientos y habilidades en su desempeño profesional, ya sea para orientar a compañeros, dar solución a problemas, o mejorar su competencia sindical.
\end{abstract}

\section{Palabras clave}

Formación online, evaluación de la formación, transferencia del aprendizaje, aprendizaje adulto.

\begin{abstract}
In this article, we provide a real example of online training assessment, focusing closely on learning transfer. The research consisted of an online survey conducted amongst 471 participants from Spain who had previously undergone online training developed by ISTAS Foundation (Instituto Sindical de Trabajo, Ambiente y Salud). Participants were surveyed on their training experience. A psychometric, descriptive and correlational analysis was performed. Questionnaire reliability was determined using Cronbach's alpha and construct validity through Exploratory Factor Analysis (EFA) by Principal Component Analysis (PCA). Qualitative and quantitative data analysis allowed us to confirm positive outcomes in every factor under scrutiny (instructional design, tutor performance, learning transfer, timing and design of the virtual environment). We were also able to distinguish various transfer levels based on criteria laid down at the beginning of the study, such as experience in the labour sector, company type and student motivations for taking the course. Learning transfer was moderate, and materializes especially in the application of knowledge and skills in participants' professional performance, either to guide peers, solve problems or improve their union work.
\end{abstract}

\section{Key words}

Online courses, course evaluation, transfer of training, adult learning. 


\section{Introducción}

El e-learning es hoy en día un elemento clave en una amplia variedad de contextos formativos y educativos. Está presente en el corazón de muchos planes de negocio, considerando que las tecnologías proporcionan un nuevo escenario de herramientas que pueden agregar valor a los modos tradicionales de aprendizaje, tales como la accesibilidad a los contenidos, la gestión eficiente de cursos y alumnos, y el canal de implementación mejorado (Joo, Lim, \& Kim, 2012). Así, la evaluación de la formación de cursos online ha sido trabajada en los últimos quince años gracias al impacto de este tipo de formación e-Learning. A esto ha contribuido la inquietud por establecer estándares que permitan certificar la calidad de la formación. Ya hace una década que Rubio (2003) exponía dos enfoques de esta evaluación: el parcial, que atiende a la actividad formativa, a los materiales de formación, a las plataformas o a la relación coste/beneficio; y el global, con dos tendencias, los sistemas de evaluación centrados en modelos y/o normas de calidad estándar y calidad total, y los sistemas basados en la práctica del Bechmarking. Este último es un proceso que permite a un centro u organización compararse con otro que obtiene resultados excelentes de calidad, con el fin de emularlo.

El modelo de evaluación de acciones formativas presentado por Kirkpatrick (2004) asume cuatro niveles interconectados, que pueden ser reconocidos en e-Learning: a) reacción de los participantes ante la formación recibida; b) aprendizaje: grado de cambio de actitudes, ampliación de conocimientos y mejora de habilidades; c) cambios en la conducta o transferencia del aprendizaje; y d) resultados finales.

Tello (2010) crea un modelo de evaluación de la calidad de los cursos formativos impartidos a través de Internet, denominado MEPFI, partiendo de esos cuatro niveles de Kirkpatrick, a los que une las características internas del programa de formación mencionadas por Marquès (1999) en su plantilla de evaluación. Esta se compone de criterios de calidad sobre: aspectos técnicos y estéticos (elementos del programa), aspectos pedagógicos (indicios o impacto del aprendizaje) y aspectos funcionales (eficacia, eficiencia y relevancia).

Para los aspectos pedagógicos, Marquès (1999) valora los siguientes: plan docente (objetivos claros y explícitos), motivación (contenidos, actividades y materiales atractivos), relevancia de los elementos multimedia, guías didácticas y ayudas útiles, flexibilización del aprendizaje (tutorización, progresión de las actividades y profundidad de los contenidos), orientación del usuario, tutorización de los itinerarios, autonomía del estudiante (toma de decisiones, autoevaluación, autoaprendizaje), potencialidad y multiplicidad de los recursos didácticos, múltiples actividades formativas, enfoque crítico, aplicativo, creativo junto al aprendizaje colaborativo para la construcción de conocimiento, feedback inmediato (informes), adecuación a las características de los destinatarios, evaluación de los aprendizajes (pruebas, sistema de seguimiento para facilitar el autocontrol del trabajo) y sistema de apoyo docente y tutorial.

Tran et al. (2014) desarrollaron el perfil de resultados del estudiante e-Learning vietnamita (VESOP, por sus siglas en inglés) con objeto de evaluar el impacto de los métodos de aprendizaje sobre los resultados de aprendizaje de los estudiantes en cursos online sobre salud pública. Lograron reducir los ítems a cinco dominios: destrezas

La evaluación de cursos de formación online: el caso ISTAS. Javier Rodríguez-Santero y Juan Jesús Torres-Gordillo. 
académicas, habilidades intelectuales, interacción, responsabilidad y compromiso educativo. Solo la interacción mostró un moderado incremento. La interacción viene a ser uno de los objetivos principales de la formación e-Learning (Perera-Rodríguez, 2007).

El estudio de Sun, Tsai, Finger, Chen, \& Yeh (2008) reveló siete factores críticos que afectan a la satisfacción percibida por los estudiantes en e-Learning: la ansiedad ante el ordenador del estudiante, la actitud del tutor de e-Learning, la flexibilidad del curso, la calidad del curso, la utilidad percibida, la facilidad de uso percibida, y la diversidad en las evaluaciones. La retroalimentación ayuda a definir o identificar las metas de aprendizaje, mejorar los resultados (feedback) y autorregular el propio aprendizaje (feedforward) (García-Jiménez, 2015). En la mejora de la evaluación del aprendizaje en formación online ya existen tecnologías de la web semántica, como la herramienta OeLE, que permite realizar pruebas escritas con feedback ad hoc en exámenes online empleando ontologías (Sánchez-Vera, Prendes-Espinosa y Fernández-Breis, 2013). El apoyo en la gestión percibida por los empleados y el apoyo en el trabajo tienen un impacto significativo en la utilidad percibida del e-Learning para el aprendizaje individual, y el apoyo organizacional percibido tiene una influencia significativa en la utilidad percibida del e-Learning para el aprendizaje social (Cheng, Wang, Moormann, Olaniran, \& Chen, 2012). Niveles más altos de conocimiento retenido y el apoyo al compañero de trabajo incrementan la transferencia del aprendizaje (Homklin, Takahashi, \& Techakanont, 2014).

Bausch, Michel, \& Sonntag (2014) estudiaron cómo el género influye en el efecto de la edad sobre la autoeficacia y el éxito de la formación. Encontraron que las mujeres de mayor edad mostraron desarrollos más positivos en estos dos aspectos en comparación con los hombres de más edad. Wang y Newlin (2002) concluyen que los estudiantes con mayor autoeficacia son más proclives a adoptar el aprendizaje basado en la red y obtener significativamente mejores calificaciones finales.

Por otro lado, también existen investigaciones que muestran estudiantes poco satisfechos con la calidad de la formación, como la experiencia con cursos de Educación Social de la Universidad Nacional de Educación a Distancia en España (Santoveña, 2007). Las necesidades no cubiertas al final de la formación se relacionan con la atención por parte de los docentes, el filtro de los mensajes en los foros, la calidad de los materiales didácticos o los recursos disponibles, entre otras. De igual forma, solicitan funciones de apoyo, orientación y motivación desde un correcto uso docente de las herramientas de comunicación de la plataforma (Santoveña, 2007).

No obstante, un aspecto en el que coinciden la mayoría de estudios hoy en día y que viene a dar sentido global a la calidad de la formación online es la implicación de los estudiantes en todas las actividades formativas (García-Valcárcel y Tejedor, 2011), incluyendo su participación en la evaluación del aprendizaje (García-Jiménez, 2015), desde un diseño didáctico interactivo y colaborativo en red (Santos y Silva, 2009). Los esfuerzos actuales deben llevarnos a ayudar al estudiante a emplear las TIC para la construcción de su propio conocimiento (Centeno y Cubo, 2013), alineado a un aprendizaje profundo. Los enfoques de aprendizaje juegan un papel relevante en la calidad de la formación, donde las valoraciones positivas de las TIC se relacionan

La evaluación de cursos de formación online: el caso ISTAS. Javier Rodríguez-Santero y Juan Jesús Torres-Gordillo. 
positivamente con un enfoque profundo de aprendizaje (Maquilón, Mirete, GarcíaSánchez y Hernández-Pina, 2013).

La finalidad general de esta investigación es conocer el nivel de adecuación de los cursos de formación online propuestos por ISTAS (Instituto Sindical de Trabajo, Ambiente y Salud), así como el grado de satisfacción y el nivel de transferencia real que han supuesto a los participantes. Los objetivos específicos podrían resumirse en conocer las propiedades psicométricas del instrumento de recogida de datos empleado, las valoraciones de los participantes con respecto a cada uno de los elementos del diseño formativo (diseño pedagógico, actuación del tutor, temporalización, diseño del entorno virtual y transferencia del aprendizaje), la transferencia de las acciones formativas (entendida como cambios positivos en el quehacer profesional) y las relaciones que esta guarda con variables como el 'género', la 'antigüedad en el sector laboral', el 'cargo que se desarrolla en la empresa', el 'curso recibido', la 'tipología de empresa a la que se pertenece' y el 'motivo por el cual se realizó el curso'.

ISTAS viene ejecutando, desde antes del año 2000, cursos de formación online sobre salud laboral y medio ambiente, para CC.OO., en los planes intersectoriales, sectoriales y territoriales del ámbito nacional. No obstante, hasta la fecha de inicio de este proyecto de investigación no había efectuado una evaluación sistemática de las acciones formativas que desarrollaba, por lo que desconocía cuál era la transferencia real de las mismas sobre el alumnado que las recibía. La investigación se centró en evaluar todas las acciones formativas desarrolladas por la institución, a través de su plataforma de eLearning. ISTAS es una fundación autónoma que tiene el objetivo general de impulsar actividades de progreso social para la mejora de las condiciones de trabajo, la protección del medio ambiente y la promoción de la salud de los trabajadores.

\section{Método}

Para llevar a cabo esta investigación se recurre a una metodología no experimental, más concretamente, un estudio de encuesta.

\section{Participantes}

Se ha llevado a cabo un muestreo no probabilístico accidental, muy común en este tipo de investigaciones (Bisquerra, 2004; Grande \& Abascal, 2005; McMillan \& Schumacher, 2005), que ha dado lugar a una muestra final de 471 participantes. De haber podido seleccionar los sujetos al azar y tomando en consideración que la población de alumnos que ha recibido cursos de formación online, a través de la fundación ISTAS, asciende a 1769, estaríamos ante un tamaño muestral estadísticamente representativo para un nivel de confianza del 95\%, un error inferior al $\pm 4 \%$ y $\mathrm{P}=\mathrm{Q}$.

Con respecto a las características demográficas de la muestra (ver Tabla 1), el 54,1\% son hombres y el 45,9\% mujeres, con una edad media que ronda los 40 años (media 40,5, desviación típica 7,9). Predominan los cargos de tipo operativo $(39,3 \%)$ y de mando intermedio $(38,6 \%)$, pertenecientes mayoritariamente $(63,7 \%)$ a empresas con más de 250 trabajadores que han realizado sobre todo el curso superior (34\%) o el de riesgos psicosociales en el entorno laboral (20\%). La antigüedad laboral es muy

La evaluación de cursos de formación online: el caso ISTAS. Javier Rodríguez-Santero y Juan Jesús Torres-Gordillo. 
diversa, por lo que puede entenderse que estos resultan de utilidad, tanto para aquellos que necesitan adquirir nuevos conocimientos, como para quienes desean reciclar los que ya poseen. De hecho, estos son dos de los aspectos más señalados, con un 40,6\% y un $18,7 \%$ respectivamente, cuando se les preguntó por los motivos que les llevaron a realizar los cursos.

\section{Tabla 1}

Características de los participantes

\begin{tabular}{|c|c|c|c|c|c|c|}
\hline Género & $\begin{array}{c}\text { Femenino } \\
(45,9 \%)\end{array}$ & $\begin{array}{l}\text { Masculino } \\
(54,1 \%)\end{array}$ & & & & \\
\hline Edad & $\begin{array}{c}22 \text { a } 30 \text { años } \\
(11 \%)\end{array}$ & $\begin{array}{c}31 \text { a } 40 \text { años } \\
(37,8 \%)\end{array}$ & $\begin{array}{c}41 \text { a } 50 \text { años } \\
(41,4 \%)\end{array}$ & $\begin{array}{l}\text { Más de } 50 \\
(9,8 \%)\end{array}$ & & \\
\hline $\begin{array}{l}\text { Antigüedad } \\
\text { en el sector }\end{array}$ & $\begin{array}{c}0 \text { a } 9 \text { años } \\
(31,8 \%)\end{array}$ & $\begin{array}{c}10 \text { a } 15 \text { años } \\
(23,6 \%)\end{array}$ & $\begin{array}{c}16 \text { a } 20 \text { años } \\
(17,6 \%)\end{array}$ & $\begin{array}{c}\text { Más de } 20 \text { años } \\
(27 \%)\end{array}$ & & \\
\hline $\begin{array}{l}\text { Cargo } \\
\text { desarrollado }\end{array}$ & $\begin{array}{l}\text { Ejecutivo } \\
(4,9 \%)\end{array}$ & $\begin{array}{c}\text { Cargo intermedio } \\
(38,6 \%)\end{array}$ & $\begin{array}{c}\text { Operativo } \\
(39,3 \%)\end{array}$ & $\begin{array}{c}\text { Auxiliares } \\
\text { administrativos } \\
(17,2 \%)\end{array}$ & & \\
\hline $\begin{array}{l}\text { Tipología de } \\
\text { empresa }\end{array}$ & $\begin{array}{c}\text { Gran empresa } \\
\quad(63,7 \%)\end{array}$ & $\begin{array}{c}\text { Medianas } \\
\text { empresas } \\
(18,9 \%)\end{array}$ & $\begin{array}{c}\text { Pequeñas } \\
\text { empresas } \\
(11,9 \%)\end{array}$ & $\begin{array}{c}\text { Microempresas } \\
(5,5 \%)\end{array}$ & & \\
\hline $\begin{array}{l}\text { Curso } \\
\text { recibido }\end{array}$ & $\begin{array}{c}\text { Sistema de } \\
\text { Gestión } \\
\text { Medioambiental } \\
(3,8 \%)\end{array}$ & $\begin{array}{c}\text { Riesgos } \\
\text { psicosociales en el } \\
\text { entorno laboral } \\
(20 \%)\end{array}$ & $\begin{array}{l}\text { Superior } \\
(34 \%)\end{array}$ & $\begin{array}{l}\text { Básico } 80 \mathrm{H} \\
\quad(7,6 \%)\end{array}$ & $\begin{array}{l}\text { Intervención } \\
\text { sindical } \\
(8,7 \%)\end{array}$ & $\begin{array}{c}\text { Otros } \\
(25,9 \%)\end{array}$ \\
\hline $\begin{array}{l}\text { Motivo } \\
\text { realización } \\
\text { curso }\end{array}$ & $\begin{array}{c}\text { Adquirir } \\
\text { conocimientos } \\
(40,6 \%)\end{array}$ & $\begin{array}{c}\text { Actualizar } \\
\text { conocimientos } \\
(18,7 \%)\end{array}$ & $\begin{array}{c}\text { Obtención de } \\
\text { certificación } \\
\quad(5,3 \%)\end{array}$ & $\begin{array}{c}\text { Mejora en la } \\
\text { situación laboral } \\
\quad(13,4 \%)\end{array}$ & $\begin{array}{c}\text { Mejora en la } \\
\text { acción } \\
\text { sindical } \\
(20,6 \%) \\
\end{array}$ & $\begin{array}{l}\text { Otros } \\
(1,4 \%)\end{array}$ \\
\hline
\end{tabular}

\section{Instrumento}

Para la recogida de datos se empleó un cuestionario online que aglutinaba 53 preguntas, de las cuales, 36 eran ítems de respuesta cerrada que recababan información en torno a las cinco dimensiones objeto de estudio (diseño pedagógico, actuación del tutor, transferencia del aprendizaje, temporalización y diseño del entorno virtual). El resto de ítems recogía información sobre características de la muestra y las opiniones de los sujetos, a través de preguntas con respuesta abierta.

\section{Procedimiento}

El enlace al cuestionario online fue enviado, a través de correo electrónico, a los 1769 alumnos que habían recibido, meses antes, los cursos de la fundación ISTAS. Los 471 que lo cumplimentaron constituyeron la muestra participante. Gracias a la administración online, no solo garantizamos el anonimato de las respuestas, sino que además se contó con la matriz de datos de manera prácticamente inmediata, lo que agilizó, en gran medida, los análisis necesarios para responder a los objetivos de la investigación.

\section{Análisis de datos}

La evaluación de cursos de formación online: el caso ISTAS. Javier Rodríguez-Santero y Juan Jesús Torres-Gordillo. 
Se ha efectuado un análisis psicométrico, descriptivo y correlacional. La fiabilidad del instrumento se determinó mediante la prueba Alfa de Cronbach y la validez de constructo a través del Análisis Factorial Exploratorio (AFE) por componentes principales (ACP). Esta técnica se aplicó tras haber descartado previamente, mediante la prueba KMO (Kaiser, Meyer y Olkin) y el test de esfericidad de Bartlett, que las correlaciones entre los ítems constituyesen una matriz de identidad, lo que desaconsejaría totalmente el uso de análisis factoriales. Para la rotación de los componentes se utilizó el método Equamax, que minimiza tanto el número de número de factores como el de variables con cargas altas dentro de cada factor, mejorando así la capacidad de interpretación. Únicamente se aceptaron valores $\mathrm{R}$ de Pearson $>0.4$ (Floyd \& Widaman, 1995). Para el análisis de los datos referentes a las valoraciones sobre los cursos se ha recurrido a porcentajes, medias y desviaciones típicas, en el caso de las preguntas de respuesta cerrada, y al análisis de contenido para las abiertas. Para el estudio de las relaciones entre la transferencia y determinadas características profesionales de los participantes hemos recurrido al coeficiente de contingencia.

\section{Resultados}

Los resultados se exponen divididos en tres subapartados. En el inicial se darán a conocer propiedades psicométricas del instrumento empleado para la recogida de datos; en el segundo, se presentarán las valoraciones de los participantes respecto al grado de adecuación de los distintos elementos del diseño de las acciones formativas y el nivel de transferencia; y, en el final, profundizaremos en la relación que guarda la transferencia del aprendizaje de los cursos con variables como el 'género', 'cargo que se desarrolla en la empresa', 'antigüedad en el sector laboral', 'curso recibido', 'tipología de empresa a la que se pertenece' y 'motivo por el cual se realizó el curso'.

\section{Propiedades psicométricas del instrumento}

En cuanto a la validez de constructo del cuestionario, los resultados de la prueba KMO (.97) y del test de esfericidad de Bartlett $(p=.000)$ muestran la pertinencia de desarrollar el AFE. Tras la aplicación de este, los ítems quedan agrupados en 5 factores (diseño pedagógico, actuación del tutor, transferencia del aprendizaje logrado en el curso, temporalización y diseño del entorno virtual) con un poder explicativo de la varianza total del $67.62 \%$. En la rotación Equamax, los ítems obtuvieron pesos iguales o mayores a un valor R de .40 en el factor al que pertenecían. En cuanto a la fiabilidad, tomando como referencia los criterios de George y Mallery (2003), resultaría buena, excelente en algunos casos, puesto que se alcanzan valores alfa de Cronbach entre .82 y .96 para cada uno de los factores (ver Tabla 2).

Tabla 2

Psicometría del instrumento de medida

\begin{tabular}{lcc}
\hline \multicolumn{1}{c}{ Factor } & Ítems & Fiabilidad \\
\hline Diseño pedagógico & $1-17$ & .961 \\
Actuación del tutor & $18-23$ & .914 \\
Transferencia & $24-29$ & .819
\end{tabular}

La evaluación de cursos de formación online: el caso ISTAS. Javier Rodríguez-Santero y Juan Jesús Torres-Gordillo. 


\begin{tabular}{rcc} 
Temporalización & $30-32$ & .892 \\
Diseño del entorno virtual & $33-36$ & .899 \\
TOTAL & (todos los ítems) & .970 \\
\hline
\end{tabular}

Para futuras aplicaciones, sería recomendable emplear una versión reducida del instrumento en la que se eliminasen (ver tabla 3) los ítems con cargas factoriales considerables en más de un factor, los que no saturan convenientemente en ninguno de ellos $(\mathrm{R}<0.515)$ y el que supone una valoración general sobre la formación recibida (ítem 17), pues, en realidad, no debería vincularse a ninguno de los factores. Los 24 ítems restantes quedarían agrupados en los mismos 5 factores ya indicados, pero el poder explicativo de la varianza aumentaría al $71.58 \%$, siendo superior a 5 el porcentaje explicado por cada factor. Además, se obtendría un reparto más equitativo de los ítems en cada componente. También deberíamos plantearnos la posible eliminación del ítem 29 (El curso recibido contribuyó a mi mejora profesional) en aquellos casos en los que la evaluación se realice justo después de haber concluido la actividad formativa, pues las respuestas se corresponderán más con un deseo que con un dato empírico.

\section{Tabla 3}

Ítems a eliminar en la versión definitiva del instrumento

\begin{tabular}{|c|c|c|c|c|c|}
\hline & \multicolumn{5}{|c|}{ Componente } \\
\hline & 1 & 2 & 3 & 4 & 5 \\
\hline 5. Los contenidos cubrieron mis necesidades profesionales & .614 & & & & .416 \\
\hline $\begin{array}{l}\text { 9. La selección de los materiales empleados en el curso resultó } \\
\text { adecuada }\end{array}$ & .504 & & & & \\
\hline $\begin{array}{l}\text { 11. Los materiales empleados en el curso fueron lo suficientemente } \\
\text { didácticos }\end{array}$ & .480 & & & & \\
\hline $\begin{array}{l}\text { 12. El proceso de evaluación desarrollado fue útil para fomentar el } \\
\text { aprendizaje de los participantes del curso }\end{array}$ & & .438 & & .527 & \\
\hline $\begin{array}{l}\text { 13. Los criterios de evaluación empleados en el curso fueron } \\
\text { coherentes con las competencias programadas }\end{array}$ & & .416 & & .510 & \\
\hline 14. La información que recibí en el curso me pareció muy abundante & .439 & & .459 & & \\
\hline $\begin{array}{l}\text { 15. Los contenidos del curso respondieron a mis necesidades } \\
\text { formativas }\end{array}$ & .546 & & & & .482 \\
\hline $\begin{array}{l}\text { 16. El sistema de aprendizaje implícito en el curso logró satisfacer mis } \\
\text { necesidades }\end{array}$ & .478 & & & & .401 \\
\hline 17. Estoy satisfecho con la formación que recibí & .580 & & & & \\
\hline $\begin{array}{l}\text { 19. Los procedimientos de evaluación empleados en el curso fueron } \\
\text { suficientes para la consecución de las competencias programadas }\end{array}$ & & .537 & & .446 & \\
\hline $\begin{array}{l}\text { 20. Los criterios de calificación y ponderación fueron coherentes con } \\
\text { los conocimientos y tareas desarrollados }\end{array}$ & & .532 & & .414 & \\
\hline
\end{tabular}

La evaluación de cursos de formación online: el caso ISTAS. Javier Rodríguez-Santero y Juan Jesús Torres-Gordillo. 
24. La metodología empleada promovió el desarrollo de actitudes y

formas de comportamiento adecuadas en tu sector profesional

En cuanto a la fiabilidad que obtendríamos con esta versión reducida del instrumento (24 ítems), continuaría siendo bastante buena, puesto que se alcanzan valores alfa de Cronbach entre .78 y .94 para cada uno de los factores (ver Tabla 4).

\section{Tabla 4}

Psicometría del instrumento de medida (versión reducida)

\begin{tabular}{lcc}
\hline \multicolumn{1}{c}{ Factor } & Ítems & Fiabilidad \\
\hline Diseño pedagógico & $1-8,10$ & .935 \\
Actuación del tutor & $18,21-23$ & .914 \\
Transferencia & $25-29$ & .783 \\
Temporalización & $30-32$ & .892 \\
Diseño del entorno virtual & $33-36$ & .901 \\
& & .949 \\
\hline
\end{tabular}

\section{Valoraciones de los participantes}

Las opiniones de los participantes (ver Tabla 5) cuentan con puntuaciones medias, obtenidas para cada factor, entre 2.8 y 3.35 , con desviaciones típicas entre .5 y .7 , lo que implica valoraciones muy positivas, tomando en consideración que los valores de respuesta oscilan entre 1 (totalmente en desacuerdo) y 4 (totalmente de acuerdo).

\section{Tabla 5}

Valoraciones de los participantes (estadísticos descriptivos)

\begin{tabular}{|c|c|c|c|c|c|c|c|}
\hline & \multirow{2}{*}{ Ítems } & \multicolumn{4}{|c|}{ PORCENTAJES } & \multirow[b]{2}{*}{ M } & \multirow[b]{2}{*}{ DT } \\
\hline & & T.D. & D. & A. & T.A. & & \\
\hline 1. & Los objetivos desarrollados cubrieron mis necesidades. & 2.1 & 2.5 & 61.8 & 33.7 & 3.27 & .61 \\
\hline 2. & Los objetivos del curso se han cubierto en su totalidad. & 1.7 & 3.5 & 62.8 & 32.0 & 3.25 & .60 \\
\hline 3. & Los contenidos del curso respondieron a los objetivos propuestos. & 1.2 & 2.9 & 64.7 & 31.2 & 3.26 & .57 \\
\hline 4. & Existió una clara coordinación entre los contenidos. & .6 & 4.3 & 62.2 & 32.9 & 3.27 & .57 \\
\hline 5. & Los contenidos cubrieron mis necesidades profesionales. & 2.1 & 7.6 & 67.4 & 22.9 & 3.11 & .61 \\
\hline 6. & Los contenidos se presentaron de forma clara. & 1.4 & 7.2 & 58.5 & 32.9 & 3.23 & .64 \\
\hline 7. & $\begin{array}{l}\text { La metodología empleada en el curso fue adecuada para la } \\
\text { adquisición de las competencias programadas. }\end{array}$ & 1.4 & 7.4 & 60.7 & 30.4 & 3.20 & .63 \\
\hline 8. & $\begin{array}{l}\text { La metodología empleada englobó la aplicación de los } \\
\text { conocimientos adquiridos. }\end{array}$ & 1.7 & 7.0 & 65.9 & 25.4 & 3.15 & .61 \\
\hline 9. & $\begin{array}{l}\text { La selección de los materiales empleados en el curso resultó } \\
\text { adecuada. }\end{array}$ & 1.0 & 8.7 & 64.5 & 25.8 & 3.15 & .60 \\
\hline
\end{tabular}

La evaluación de cursos de formación online: el caso ISTAS. Javier Rodríguez-Santero y Juan Jesús Torres-Gordillo. 
10. Los materiales del curso fueron útiles y ofrecieron información adecuada.

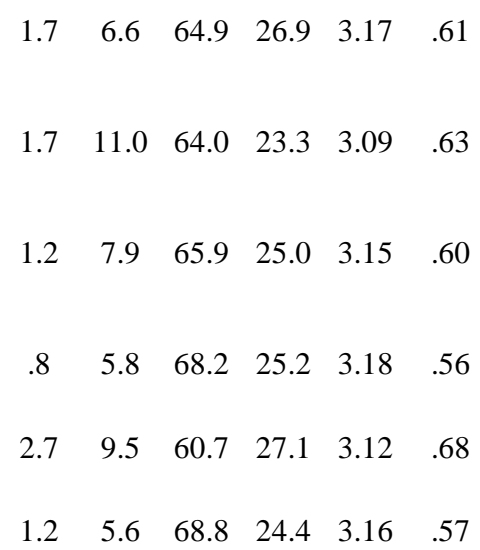

$\begin{array}{llllll}1.7 & 11.0 & 64.0 & 23.3 & 3.09 & .63\end{array}$

$\begin{array}{llllll}1.2 & 7.9 & 65.9 & 25.0 & 3.15 & .60\end{array}$

$\begin{array}{llllll}.8 & 5.8 & 68.2 & 25.2 & 3.18 & .56\end{array}$

$\begin{array}{llllll}2.7 & 9.5 & 60.7 & 27.1 & 3.12 & .68\end{array}$

$\begin{array}{llllll}1.2 & 5.6 & 68.8 & 24.4 & 3.16 & .57\end{array}$

11. Los materiales empleados en el curso fueron lo suficientemente didácticos.

12. El proceso de evaluación desarrollado fue útil para fomentar el aprendizaje de los participantes del curso.

13. Los criterios de evaluación empleados en el curso fueron coherentes con las competencias programadas.

14. La información que recibí en el curso me pareció abundante.

15. Los contenidos del curso respondieron a mis necesidades formativas.

16. El sistema de aprendizaje implícito en el curso logró satisfacer mis necesidades.

$\begin{array}{llllll}1.2 & 6.2 & 69.8 & 22.7 & 3.14 & .57\end{array}$

17. Estoy satisfecho con la formación que recibí. \begin{tabular}{llllll}
1.4 & 4.8 & 59.5 & 34.3 & 3.27 & .61 \\
\hline
\end{tabular}

18. El tutor del curso empleó los resultados de las evaluaciones para orientar el aprendizaje de los participantes del curso.

$\begin{array}{llllll}1.4 & 10.7 & 51.7 & 36.2 & 3.23 & .69\end{array}$

19. Los procedimientos de evaluación empleados en el curso fueron suficientes para la consecución de las competencias programadas.

$\begin{array}{llllll}1.2 & 7.0 & 69.6 & 22.1 & 3.13 & .57\end{array}$

20. Los criterios de calificación y ponderación fueron coherentes con los conocimientos y tareas desarrollados.

$\begin{array}{llllll}.8 & 6.2 & 70.7 & 22.3 & 3.14 & .55\end{array}$

21. La acción tutorial me orientó según mis necesidades concretas.

$\begin{array}{llllll}1.9 & 7.9 & 53.3 & 37.0 & 3.25 & .68\end{array}$

22. El tutor aclaró las dudas existentes empleando para ello todos los recursos disponibles.

$\begin{array}{llllll}2.1 & 5.0 & 49.0 & 44.0 & 3.35 & .67\end{array}$

23. La comunicación con el tutor fue fluida.

$\begin{array}{llllll}2.3 & 6.6 & 46.1 & 45.0 & 3.34 & .70\end{array}$

24. La metodología empleada promovió el desarrollo de actitudes y formas de comportamiento adecuadas en tu sector profesional.

25. La metodología desarrollada supuso la resolución de problemas reales de tu práctica profesional.

26. Los aprendizajes logrados en el curso me parecen fáciles de llevar a mi práctica cotidiana.

$\begin{array}{llllll}1.7 & 22.5 & 65.3 & 10.5 & 2.85 & .61\end{array}$

27. El curso recibido nos ha mostrado aplicaciones potenciales de los aprendizajes en nuestro ámbito profesional.

$\begin{array}{llllll}.8 & 11.6 & 73.8 & 13.8 & 3.01 & .54\end{array}$

28. Estoy aplicando o pienso aplicar parcial/totalmente los contenidos adquiridos en el curso.

$\begin{array}{llllll}.6 & 10.2 & 67.1 & 22.1 & 3.11 & .58\end{array}$

29. El curso recibido contribuyó a mi mejora profesional.

\begin{tabular}{llllll}
3.2 & 23.1 & 56.3 & 17.4 & 2.88 & .72 \\
\hline
\end{tabular}

30. La duración del curso fue la adecuada. $\quad \begin{array}{llllll}1.4 & 13.4 & 64.7 & 20.5 & 3.04 & .63\end{array}$

31. El tiempo programado en el curso para el trabajo de los contenidos fue adecuado.

$\begin{array}{llllll}1.4 & 13.4 & 65.9 & 19.2 & 3.03 & .62\end{array}$

32. El tiempo programado en el curso para la ejecución de las actividades / tareas fue el adecuado. 
33. La estructura de la plataforma me resultó clara, lógica y bien organizada.

34. Accedí con facilidad a los distintos espacios del curso.

35. Los canales de comunicación a disposición de los participantes del curso fueron los adecuados.

36. Los recursos que ofreció la plataforma fueron útiles y suficientes para gestionar mi autoaprendizaje.

En lo que respecta al diseño pedagógico de los cursos, tomando en consideración que las medias oscilan entre 3.09 y 3.27 y que las desviaciones típicas no superan el .68, podríamos afirmar que, en términos generales, el sistema de aprendizaje implícito en los cursos ha logrado satisfacer las necesidades de los participantes, gracias a una buena coordinación entre los objetivos propuestos, los contenidos desarrollados, los materiales empleados y el sistema de evaluación utilizado.

En las manifestaciones sobre los objetivos, se produce una concentración de las opiniones en las opciones positivas de respuesta de más del 94\%, dando lugar a una media de 3.25 y desviación típica de 0.6. Estos datos nos permiten afirmar que los participantes en los cursos consideran, en gran medida, que los objetivos se cubren en su totalidad y que la consecución de los mismos permite satisfacer sus necesidades de partida. Por otra parte, la gran mayoría (por encima del $90 \%$ en todos los ítems) opina que los contenidos seleccionados muestran una clara coordinación entre sí, responden a los objetivos propuestos, no resultan ambiguos o farragosos y sirven para satisfacer sus necesidades profesionales de partida. Con respecto a los materiales, más del $90 \%$ opina que han resultado adecuados, útiles y didácticos. En cuanto a los procedimientos de evaluación empleados, se consideran coherentes con las competencias programadas y útiles para fomentar el aprendizaje, pues las puntuaciones medias han superado el valor 3,14 , las desviaciones típicas son relativamente bajas (.56 y .6) y el nivel de concentración de las opiniones en las opciones positivas de respuesta supera el $90 \%$.

Centrándonos en la actuación del tutor, los tutores han establecido una comunicación fluida $(\mathrm{M}=3.34$; $\mathrm{DT}=.7)$ que ha permitido aclarar dudas, empleando todos los recursos disponibles $(\mathrm{M}=3.35$; $\mathrm{DT}=.67)$, y orientar sobre las necesidades particulares $(\mathrm{M}=3.25 ; \mathrm{DT}=.68)$, tomando como referencia los resultados de las evaluaciones $(\mathrm{M}=$ 3.23; DT = .69). En este sentido, los criterios de evaluación y calificación se perciben coherentes con las tareas desarrolladas $(\mathrm{M}=3.14 ; \mathrm{DT}=.55)$, permitiendo finalmente la consecución de las competencias programadas $(\mathrm{M}=3.13$; $\mathrm{DT}=.57)$.

La temporalización de los cursos resulta bastante adecuada $(\mathrm{M}=3.04 ; \mathrm{DT}=.63)$. Por lo general, los encuestados opinan que el tiempo para trabajar sobre los contenidos $(\mathrm{M}=$ 3.04; DT $=.63)$ y realizar las tareas $(\mathrm{M}=3.04 ; \mathrm{DT}=.63)$ ha sido suficiente.

En cuanto al diseño del entorno virtual, este ha resultado claro, lógico y bien organizado $(\mathrm{M}=3.29 ; \mathrm{DT}=.64)$. Los participantes opinan, en gran medida, que han logrado gestionar convenientemente su aprendizaje gracias a los recursos disponibles $(M=3.29$; DT $=.61)$, destacando los distintos canales de comunicación empleados $(\mathrm{M}=3.32$; DT $=.64)$ y la facilidad para acceder a los distintos apartados de los cursos $(\mathrm{M}=3.35$; $\mathrm{DT}=$ $.64)$.

La evaluación de cursos de formación online: el caso ISTAS. Javier Rodríguez-Santero y Juan Jesús Torres-Gordillo. 
Respecto a la transferencia del aprendizaje, apartado central de nuestro estudio, los resultados indican una tendencia considerable a expresar que se ha contribuido a la mejora profesional $(\mathrm{M}=2.88$; $\mathrm{DT}=.72)$, no solo por haber trabajado con problemas reales de la práctica $(\mathrm{M}=2.93$; $\mathrm{DT}=.65)$ y haber mostrado aplicaciones potenciales de los aprendizajes $(\mathrm{M}=3.01$; DT $=.54)$, sino, sobre todo, porque han llegado a desarrollarse formas de comportamiento adecuadas para el sector profesional $(\mathrm{M}=3.06$; DT $=.59)$ y se están aplicando, o piensan aplicarse, los conocimientos adquiridos $(\mathrm{M}=$ 3.11 ; DT $=.58)$, pese a que ello no resulte tan fácil como desearían $(\mathrm{M}=2.85 ; \mathrm{DT}=$ .61). Tomando como referencia las respuestas de los 194 sujetos que contestaron a la pregunta abierta sobre la transferencia, podemos establecer cinco categorías de logro distintas:

- Mejora profesional (52,0\% de las manifestaciones): se refiere a la aplicación de aprendizajes, adquiridos en el curso, al trabajo que desarrollan.

“...Era necesario para mí tener la formación para realizar mi trabajo correctamente. Por lo tanto ahora puedo realizar mi trabajo más satisfactoriamente".

- Adquisición de conocimientos útiles para la práctica $(29,9 \%)$ : aglutinaría todas aquellas manifestaciones que señalan la posesión de conocimientos útiles a aplicar en el ámbito laboral, tras la realización de los cursos.

\section{“...Mayor conocimiento para afrontar situaciones que de otra manera no hubiera podido resolver".}

- Adquisición de habilidades y destrezas útiles para el trabajo (5,7\%): comentarios sobre las nuevas habilidades y destrezas potencialmente útiles, desde el punto de vista de la práctica profesional, obtenidas tras la realización del curso.

\section{“...He adquirido nuevas competencias que me dan seguridad en mi trabajo diario”.}

- Mejoras en mi situación laboral (6,7\%): engloba todas aquellas respuestas en las que se señala un ascenso o un cambio en el tipo de trabajo que se desarrolla, gracias a la titulación o a los aprendizajes obtenidos durante el curso.

“...El curso me ha servido para promocionar profesionalmente, actualmente ejerzo como técnico de prevención”.

- Mejoras a nivel personal y actitudinal (5,7\%): conjunto de respuestas que señalan cambios a nivel personal y actitudinal en el ámbito profesional, como fruto del curso realizado.

“... La formación recibida ha confirmado mis opiniones y conocimientos y ha hecho que ( $y$ es para mí importante) mi autoestima se refuerce $y$ que mi quehacer diario sea más sólido y seguro".

Relaciones entre la transferencia y determinadas características profesionales de los participantes

La evaluación de cursos de formación online: el caso ISTAS. Javier Rodríguez-Santero y Juan Jesús Torres-Gordillo. 
Para profundizar en el estudio de la transferencia hemos analizado la existencia de relaciones entre la variable 'la formación recibida ha supuesto cambios positivos en mi quehacer profesional' y las referentes al 'género', 'cargo que se desarrolla en la empresa', 'antigüedad en el sector laboral', 'curso recibido', 'tipología de empresa a la que se pertenece' y 'motivo por el cual se realizó el curso'.

Pasamos a exponer únicamente aquellos casos donde se ha constatado la existencia de relación entre las variables.

Atendiendo al nivel de significación (.000) y el valor del coeficiente de contingencia (.214) de la Tabla 6, podemos afirmar la existencia de una relación, aunque de intensidad baja, entre los años de antigüedad en el sector laboral y opinar que la formación recibida ha supuesto cambios positivos en el quehacer profesional. Tomando como base las diferencias entre los recuentos y las frecuencias observadas, concluimos que dicha relación se establece con quienes cuentan con una experiencia de más de 20 años, y, también, aunque en menor medida, con quienes llevan trabajando de 16 a 20 años, lo cual parece indicar que la transferencia del aprendizaje ha sido mayor en los más veteranos.

\section{Tabla 6}

Correlación entre los años de antigüedad en el sector laboral y la transferencia

\begin{tabular}{|c|c|c|c|c|c|}
\hline \multirow{2}{*}{$\begin{array}{c}\text { Antigüedad en el } \\
\text { sector laboral (años) }\end{array}$} & & \multicolumn{2}{|c|}{$\begin{array}{c}\text { La formación recibida ha supuesto } \\
\text { cambios positivos en mi quehacer } \\
\text { profesional }\end{array}$} & \multirow{2}{*}{$\begin{array}{l}\text { Valor del } \\
\text { coeficiente de } \\
\text { contingencia }\end{array}$} & \multirow[t]{2}{*}{ Sig. } \\
\hline & & Sí & No & & \\
\hline \multirow{2}{*}{$0-9$} & Recuento & 62 & 88 & & \\
\hline & Frecuencia. E. & 76.1 & 73.9 & & \\
\hline \multirow{2}{*}{$10-15$} & Recuento & 53 & 58 & & \\
\hline & Frecuencia. E. & 56.3 & 54.7 & .214 & $.000 * * *$ \\
\hline \multirow{2}{*}{$16-20$} & Recuento & 50 & 33 & & \\
\hline & Frecuencia. E. & 42.1 & 40.9 & & \\
\hline \multirow[t]{2}{*}{ Más de 20} & Recuento & 74 & 53 & & \\
\hline & Frecuencia. E. & 64.4 & 62.6 & & \\
\hline
\end{tabular}

Podemos afirmar también (ver Tabla 7) la existencia de una relación significativa (95\%), aunque de baja intensidad, entre trabajar en una gran empresa y opinar que la formación recibida en los cursos ha supuesto cambios positivos en el quehacer profesional, de lo que se deduce que cuanto mayor es la empresa, mejor es la transferencia lograda por los cursos.

La evaluación de cursos de formación online: el caso ISTAS. Javier Rodríguez-Santero y Juan Jesús Torres-Gordillo. 


\section{Tabla 7}

Correlación entre la tipología de empresa y la transferencia

\begin{tabular}{|c|c|c|c|c|c|}
\hline \multirow[t]{2}{*}{$\begin{array}{c}\text { Tipología de empresa a la } \\
\text { que perteneces }\end{array}$} & & \multicolumn{2}{|c|}{$\begin{array}{l}\text { La formación recibida ha } \\
\text { supuesto cambios positivos en } \\
\text { mi quehacer profesional }\end{array}$} & \multirow[t]{2}{*}{$\begin{array}{c}\text { Valor del } \\
\text { coeficiente de } \\
\text { contingencia }\end{array}$} & \multirow[t]{2}{*}{ Sig. } \\
\hline & & Sí & No & & \\
\hline Microempresa (menos 10 & Recuento & 10 & 16 & & \\
\hline trabajadores) & Frecuencia. E. & 13,2 & 12,8 & & \\
\hline Pequeña empresa (entre 10 & Recuento & 24 & 32 & & \\
\hline y 49 trabajadores) & Frecuencia. E. & 28,4 & 27,6 &, 13 &, $042 *$ \\
\hline Mediana empresa (entre 50 & Recuento & 38 & 51 & & \\
\hline y 249 trabajadores) & Frecuencia. E. & 45,2 & 43,8 & & \\
\hline Gran empresa (a partir de & Recuento & 167 & 133 & & \\
\hline 250 trabajadores) & Frecuencia. E. & 152,2 & 147,8 & & \\
\hline
\end{tabular}

Como puede observarse en la Tabla 8 , existe, igualmente, una relación significativa $(\mathrm{p}=$ .000), de intensidad baja, entre apuntarse a los cursos para mejorar la acción sindical y opinar que la formación recibida ha supuesto cambios positivos en el quehacer profesional, por lo que podríamos interpretar que es en este ámbito en el que se ha producido una mayor transferencia.

\section{Tabla 8}

Correlación entre el motivo por el que se apuntan a los cursos y el impacto

\begin{tabular}{|c|c|c|c|c|c|}
\hline \multirow[t]{2}{*}{ ¿Por qué motivo te apuntaste? } & & \multicolumn{2}{|c|}{$\begin{array}{l}\text { La formación recibida ha } \\
\text { supuesto cambios } \\
\text { positivos en mi quehacer } \\
\text { profesional }\end{array}$} & \multirow[t]{2}{*}{$\begin{array}{c}\text { Valor del } \\
\text { coeficiente de } \\
\text { contingencia }\end{array}$} & \multirow[t]{2}{*}{ Sig. } \\
\hline & & Sí & No & & \\
\hline \multirow[t]{2}{*}{ Adquirir nuevos conocimientos } & Recuento & 94 & 97 & \multirow{7}{*}{, 213} & \multirow{7}{*}{, $000 * * *$} \\
\hline & Frecuencia. E. & 96,9 & 94,1 & & \\
\hline \multirow{2}{*}{ Actualizar mis conocimientos } & Recuento & 43 & 45 & & \\
\hline & Frecuencia. E. & 44,7 & 43,3 & & \\
\hline \multirow{2}{*}{ Obtener un certificado o diploma } & Recuento & 9 & 16 & & \\
\hline & Frecuencia. E. & 12,7 & 12,3 & & \\
\hline Mejorar tu situación laboral & Recuento & 25 & 38 & & \\
\hline
\end{tabular}

La evaluación de cursos de formación online: el caso ISTAS. Javier Rodríguez-Santero y Juan Jesús Torres-Gordillo. 


\begin{tabular}{llcc}
\hline & Frecuencia. E. & 32,0 & 31,0 \\
Mejorar tu acción sindical & Recuento & 67 & 30 \\
& Frecuencia. E. & 49,2 & 47,8 \\
\multirow{2}{*}{ Otros } & Recuento & 1 & 6 \\
& Frecuencia. E. & 3,6 & 3,4 \\
\hline Nota: $* p<05 * * p<01 * * * p<001$ & &
\end{tabular}

\section{Discusión}

Si bien existen otros instrumentos para la evaluación de la formación online en el contexto nacional español, como el FET (Pineda, Ciraso y Quesada, 2014) o el Cuestionario de Factores de Transferencia Docente (Feixas et al., 2013), el que aquí presentamos no solo atiende a la transferencia del aprendizaje en el desempeño profesional, sino también a otras dimensiones relevantes en los procesos de e-Learning, como el diseño pedagógico, la actuación del tutor, la temporización o el diseño del entorno virtual. Además, es aplicable a cualquier actividad formativa, aspecto en el que nos distanciamos del de Feixas et al. (2013), centrado exclusivamente en la docencia universitaria. Quesada, Espona, Ciraso y Pineda (2015) no han hallado con el FET diferencias significativas, en cuanto al grado de transferencia, entre los trabajadores que hicieron la formación presencial y los que siguieron formación e-Learning, aunque sí difieren en el modelo explicativo de cada modalidad formativa con distintos factores y pesos de estos.

Centrándonos en los resultados de nuestra investigación, podríamos afirmar que el perfil más común entre quienes reciben los cursos de formación sería el de un sujeto de unos 40 años, que trabaja con más de 250 compañeros, desempeñando un cargo de tipo operativo o de mando intermedio, que pretende adquirir nuevos conocimientos realizando el curso superior en prevención de riesgos laborales.

Las valoraciones de los participantes en todas y cada una de las dimensiones objeto de estudio son muy positivas. Nuestros resultados corroboran los planteamientos de García-Valcárcel y Tejedor (2011) en lo referente a que la identificación, por parte de los participantes, con la forma de trabajo del tutor (objetivos, metodología, recursos...), resulta un estímulo motivador en el proceso formativo. Parafraseando a Kirkpatrick (2004), el reto no está solo en que los participantes aprendan lo que necesitan saber, sino en hacerlos reaccionar de forma favorable hacia la acción formativa que se les presenta. Pineda et al. (2014) concluyeron igualmente que la motivación para transferir y la satisfacción con la formación son dos factores fuertes de la transferencia. Los dos estudios que presentan Grohmann, Beller, \& Kauffeld (2014) señalan la importancia de la motivación para la transferencia como un enlace de mediación entre las características de la formación y la transferencia de la misma.

Los importantes recursos que las empresas invierten en la formación de sus empleados merecen la pena cuando realmente lo que han aprendido se transfiere a la práctica (Hutchins, Burke, \& Berthelsen, 2010). En este sentido, más del 50\% de los participantes en esta investigación $(50,7 \%)$ ha manifestado que el desarrollo de los cursos ha supuesto cambios positivos en su quehacer profesional. Podríamos concluir que han logrado un nivel de transferencia, al menos, moderado, similar a los resultados

La evaluación de cursos de formación online: el caso ISTAS. Javier Rodríguez-Santero y Juan Jesús Torres-Gordillo. 
obtenidos por Pineda et al. (2014). Este se ha materializado, sobre todo, en la aplicación de conocimientos y habilidades en su desempeño profesional, ya sea para orientar a compañeros, dar solución a problemas, o mejorar su competencia sindical. Aunque habría que considerar que en algunos casos supuso también una mejora laboral, gracias a la titulación obtenida, o simplemente un cambio positivo a nivel personal y actitudinal a la hora de desempeñar su puesto de trabajo.

Los tutores pueden mejorar el diseño de la transferencia de un programa de formación, por ejemplo, empleando estrategias de proalimentación que faciliten la autorregulación del aprendizaje (García-Jiménez, 2015) o incluyendo estudios de casos relacionados con la propia transferencia, que muestren cómo los participantes pueden aplicar de manera efectiva los contenidos y habilidades de la formación al volver al lugar de trabajo (Grohmann et al., 2014). Además, las tareas que permiten reflexionar sobre las soluciones a los potenciales obstáculos para una transferencia exitosa, pueden incluirse directamente en el programa de formación (Grohmann et al., 2014). No cabe duda que un elemento clave en estas prácticas evaluadoras, que ponen el foco en la transferencia, está en la incorporación del propio alumno en la evaluación de aprendizaje (GarcíaJiménez, 2015).

Tomando en consideración los distintos criterios de comparación empleados, podemos afirmar que la consecución de niveles de transferencia más altos se da en los participantes con mayor experiencia laboral, que desarrollan su labor en grandes empresas, y se inscribieron en los cursos con el firme objetivo de mejorar su acción sindical. Con el fin de incrementar la transferencia del aprendizaje, las organizaciones deben centrarse más en la creación de entornos de aprendizaje que potencien el apoyo del compañero de trabajo, al menos en el corto plazo. Sin embargo, a más largo plazo, las organizaciones deben mejorar también la calidad de otros tipos de apoyo, para aprovechar de manera más eficaz las oportunidades de transferencia del aprendizaje (Homklin et al., 2014).

Entre las principales aportaciones de este trabajo podemos destacar su utilidad como ejemplo para mostrar una evaluación real de procesos de formación online. Por otro lado, se proporciona un instrumento válido y fiable que puede ser empleado para evaluar cualquier tipo de propuesta formativa.

Como limitación más importante podríamos mencionar que, al ser un estudio de encuesta (self-report), no hemos podido efectuar comprobaciones empíricas. Únicamente concluimos a partir de las manifestaciones de los encuestados, resultando imposible medir el grado de veracidad de las mismas. Esta evaluación diferida de la transferencia de aprendizajes al puesto de trabajo tomando como base indicadores no directamente observables (Quesada et al., 2015), aunque no es lo más deseable, resulta una práctica común dentro de la comunidad científica. Por otro lado, aunque disponemos de los datos correspondientes a cada uno de los cursos, hemos efectuado únicamente una evaluación global, considerando todas las respuestas como una sola muestra, careciendo por tanto este artículo de datos descriptivos referentes a cada uno de ellos. Tomemos en consideración que esta situación, si bien no afecta al objetivo de mostrar un ejemplo sobre cómo puede efectuarse la evaluación de formación online, sí puede haber incidido en los resultados obtenidos. Otro aspecto a considerar, además de

La evaluación de cursos de formación online: el caso ISTAS. Javier Rodríguez-Santero y Juan Jesús Torres-Gordillo. 
la eliminación de los ítems comentados con anterioridad, sería invertir algunos de los que integrarán la versión reducida para controlar el fenómeno de aquiescencia.

Sería conveniente, dadas las buenas propiedades psicométricas que parece tener el instrumento de recogida de datos, realizar un análisis factorial confirmatorio de la versión reducida a 24 ítems de opinión, lo que nos permitiría, presumiblemente, confirmar, con mayor seguridad aún, su validez y fiabilidad a la hora de evaluar propuestas formativas online.

Presentación del artículo: 7 de enero de 2016

Fecha de aprobación: 23 de marzo de 2016

Fecha de publicación: 30 de abril de 2016

Rodríguez-Santero, J. y Torres-Gordillo, J. J. (2016). La evaluación de cursos de formación online: el caso ISTAS. RED. Revista de Educación a Distancia. 49(9). Consultado el (dd/mm/aaaa) en http://www.um.es/ead/red/49

\section{Referencias}

Bausch, S., Michel, A., \& Sonntag, K. (2014). How gender influences the effect of age on self-efficacy and training success. International Journal of Training and Development, 18(3), 171-187. doi:10.1111/ijtd.12027

Bisquerra, R. (Coord.) (2004). Metodología de la investigación educativa. Madrid: La Muralla.

Centeno, G. y Cubo, S. (2013). Evaluación de la competencia digital y las actitudes hacia las TIC del alumnado universitario. Revista de Investigación Educativa, 31(2), 517-536. doi:10.6018/rie.31.2.169271

Feixas, M., Durán, M. M., Fernández, I., Fernández, A., García San Pedro, M. J., Márquez, M. D. ... Lagos, P. (2013). Cómo medir la transferencia de la formación en Educación Superior?: el Cuestionario de Factores de Transferencia. Revista de Docencia Universitaria. REDU, 11(3), 219-248. Recuperado el 22 de marzo de 2016, de http://red-u.net/redu/documentos/vol11_n3_completo.pdf

Floyd, F. J., \& Widaman, K. F. (1995). Factor analysis in the development and refinement of clinical assessment instruments. Psychological Assessment, 7(3), 286299. doi:10.1037/1040-3590.7.3.286

García-Jiménez, E. (2015). La evaluación del aprendizaje: de la retroalimentación a la autorregulación. El papel de las tecnologías. RELIEVE, 21(2), art. M2. doi:10.7203/relieve.21.2.7546

García-Valcárcel, A. y Tejedor, F. J. (2011). Variables TIC vinculadas a la generación de nuevos escenarios de aprendizaje en la enseñanza universitaria. Aportes de las curvas ROC para el análisis de diferencias. Educación XX1, 14(2), 43-78.

La evaluación de cursos de formación online: el caso ISTAS. Javier Rodríguez-Santero y Juan Jesús Torres-Gordillo. 
Grande, I., \& Abascal, E. (2005). Análisis de encuestas. Madrid, España: ESIC.

George, D., \& Mallery, P. (2003). SPSS for Windows step by step: A simple guide and reference. 11.0 update. Boston: Allyn \& Bacon.

Grohmann, A., Beller, J., \& Kauffeld, S. (2014). Exploring the critical role of motivation to transfer in the training transfer process. International Journal of Training and Development, 18(2), 84-103. doi:10.1111/ijtd.12030

Homklin, T., Takahashi, Y., \& Techakanont, K. (2014). The influence of social and organizational support on transfer of training: evidence from Thailand. International Journal of Training and Development, 18(2), 116-131. doi:10.1111/ijtd.12031

Hutchins, H. M., Burke, L. A., \& Berthelsen, A. M. (2010). A missing link in the transfer problem? Examining how trainers learn about training transfer. Human Resource Management, 49, 599-618.

Joo, Y. J., Lim, K. Y., \& Kim, S. M. (2012). A model for predicting learning flow and achievement in corporate e-Learning. Educational Technology \& Society, 15(1), 313325 .

Kirkpatrick, D. L. (2004). Evaluación de acciones formativas: los cuatro niveles [Evaluating Training Programs: the four levels]. Barcelona, Spain: Epise.

Maquilón, J. J., Mirete, A. B., García-Sánchez, F. A., y Hernández-Pina, F. (2013). Valoración de las TIC por los estudiantes universitarios y su relación con los enfoques de aprendizaje. Revista de Investigación Educativa, 31(2), 537554. doi:10.6018/rie.31.2.151891

Marquès, P. (1999, 12 enero). Entornos formativos multimedia: elementos, plantillas de evaluación/criterios de calidad. [Archivo en línea]. Recuperado el 28 de noviembre de 2015, de http://www.peremarques.net/calidad.htm

McMillan, J. H., \& Schumacher, S. (2005). Investigación Educativa. Madrid, España: Pearson Addison Wesley.

Perera-Rodríguez, V. H. (2007). Estudio de la interacción didáctica en e-Learning. (Tesis doctoral). Universidad de Sevilla. Sevilla. Recuperado de http://fondosdigitales.us.es/tesis/tesis/703/estudio-de-la-interaccion-didactica-en-eLearning.

Pineda, P., Ciraso, A., y Quesada, C. (2014). ¿Cómo saber si la formación genera resultados? El modelo FET de evaluación de la transferencia. Capital Humano, 292, 74-80. Recuperado el 22 de marzo de 2016, de http://factorhuma.org/attachments_secure/article/11261/c427_el_modelo_fet.pdf 
Quesada, C., Espona, B., Ciraso, A., y Pineda, P. (2015). La eficacia de la formación de los trabajadores de la Administración pública española: comparando la formación presencial con el e-Learning. Revista del CLAD Reforma y Democracia, 61, 107-132. Recuperado el 22 de marzo de 2016, de http://eprints.whiterose.ac.uk/88166/1/CLAD-2015__La\%20eficacia\%20de\%201a\%20formacion $\% 20 \mathrm{de} \% 201$ os $\% 20$ trabajadores $\% 20 \mathrm{de} \%$ 20la\%20AP\%20espanola.\%20eLearning.pdf

Rubio, M. J. (2003). Enfoques y modelos de evaluación del e-Learning. Revista Electrónica de Investigación y Evaluación Educativa (RELIEVE), 9(2). Recuperado de http://www.uv.es/RELIEVE/v9n2/RELIEVEv9n2_1.htm

Sánchez-Vera, M. M., Prendes-Espinosa, M. P., y Fernández-Breis, J. T. (2013). Tecnologías semánticas para la evaluación en red: análisis de una experiencia con la herramienta OeLE. Revista de Investigación Educativa, 31(2), 447464. doi:10.6018/rie.31.2.116721

Santos, E. y Silva, M. (2009). O desenho didático interativo na educação online. Revista Iberoamericana de Educación, 49, 267-287.

Santoveña, S. M. (2007). Análisis de cursos de Educación Social en entornos virtuales de aprendizaje y su influencia en la calidad. Enseñanza, 25, 77-90.

Sun, P. -C., Tsai, R. J., Finger, G., Chen, Y. -Y, \& Yeh, D. (2008). What drives a successful e-Learning? An empirical investigation of the critical factors influencing learner satisfaction. Computers \& Education, 50(4), 11831202. doi:10.1016/j.compedu.2006.11.007

Tello, I. (2010). Modelo de evaluación de la calidad de cursos formativos impartidos a través de Internet. Revista Iberoamericana de Educación a Distancia (RIED), 13(1), 209-240.

Tran, B. X., Nguyen, Q. L. T., Nong, V. M., Maher, R. M., Nguyen, A. T., Nguyen, H. A. ... Le, H. T. (2014). Student-centred outcomes of an e-Learning course on public health in Hanoi and New York. European Journal of Open, Distance and ELearning, 17(2), 1-14.

Wang, A. Y., \& Newlin, M. H. (2002). Predictors of web-student performance: the role of self-efficacy and reasons for taking an online class. Computers in Human Behavior, 18, 151-163.

La evaluación de cursos de formación online: el caso ISTAS. Javier Rodríguez-Santero y Juan Jesús Torres-Gordillo. 